\title{
Fractal analysis of ULF electromagnetic emissions in possible association with earthquakes in China
}

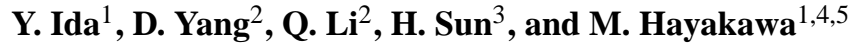 \\ ${ }^{1}$ Research Station on Seismo Electromagnetics, The University of Electro-Communications (UEC), 1-5-1 Chofugaoka, \\ Chofu Tokyo 182-8585, Japan \\ ${ }^{2}$ Institute of Geophysics, China Earthquake Administration, Beijing 100081, China \\ ${ }^{3}$ Kashi observatory, Earthquake Administration of Xinjiang Uygur Autonomous Region, Xinjiang 844000, China \\ ${ }^{4}$ Hayakawa Institute of Seismo-Electromagnetics, Co. Ltd., UEC Incubation Center, Chofu Tokyo, Japan \\ ${ }^{5}$ Earthquake Analysis Laboratory, Information Systems Inc., Kita-aoyama 2-12-42-305, Minato-ku, Tokyo 107-0061, Japan
}

Correspondence to: M. Hayakawa (hayakawa@whistler.ee.uec.ac.jp)

Received: 10 February 2012 - Revised: 22 June 2012 - Accepted: 25 September 2012 - Published: 19 October 2012

\begin{abstract}
The long-term data (during the period of 1 March 2003 through 31 December 2006) of ULF geomagnetic variations observed at Kashi station (geographic coordinates: $39.5^{\circ} \mathrm{N}, 76.0^{\circ} \mathrm{E}$ ) in China have been used to investigate the long-term variation of fractal dimension of ULF emissions. We have studied the changes in fractal dimension in association with several earthquakes around the observation station. It is then found that a significant change (or decrease) in the fractal dimension of the $Z$ component took place before the 1 September 2003 earthquake, which lends a further support to our previous finding based on our improved polarization analysis for the same earthquake. The results obtained are discussed in the contexts of a few aspects (detectability of seismogenic emissions, comparison with previous results by other analysis methods, the importance of fractal analysis in the nonlinear process of the lithosphere and earthquake prediction).
\end{abstract}

\section{Introduction}

There has been recently accumulated a lot of evidence on electromagnetic emissions in a wide frequency range associated with earthquakes (EQs) (e.g. Hayakawa and Molchanov, 2002; Molchanov and Hayakawa, 2008; Hayakawa, 2009). The DC geoelectric signals have been extensively studied mainly in Greece (Varotsos, 2005) since the 1980s. We think that the lowest AC frequency range of ULF (ultra-low frequency, with frequency less than $10 \mathrm{~Hz}$ ) is of immense importance in short-term EQ prediction. Recently, FraserSmith (2009) has summarized the evidence for ULF electromagnetic field fluctuations preceding large EQs $(M \sim 7$ or greater) including the 1964 Great Alaska EQ $(M=9.2)$ (Moore, 1964), the 1988 Spitak EQ $(M=6.9)$ (Molchanov et al., 1992; Kopytenko et al., 1993), the 1989 Loma Prieta EQ ( $M=7.1)$ (Fraser-Smith et al., 1990), and the 1993 Guam EQ $(M=7.7)$ (Hayakawa et al., 1996). He has then pointed out that any magnetic fields originating in the Earth must necessarily differ fundamentally from the ever-present and extensively studied ULF magnetic field fluctuations known as geomagnetic pulsations, which originate in the upper atmosphere (or magnetosphere) and present an obstacle in the detection of any EQ-related fluctuations. Though there have been published few papers criticizing the presence of seismogenic emissions for the Loma Prieta and Guam EQs (Campbell, 2009; Thomas et al., 2009a, b), they were not so convincing because they made just the eyeball comparison of the anomaly with geomagnetic activity. Some more positive evidence on the presence of ULF events for other EQs has been summarized in Hattori (2004) and Hayakawa et al. (2007).

The serious problem regarding these seismogenic ULF emissions is how to detect these weak signals as an EQ precursor. There have been developed different kinds of analysis methods to detect such seismogenic ULF emissions: (1) polarization analysis by means of the ratio of vertical magnetic field component to the horizontal (Hayakawa et al., 1996), (2) fractal analysis (mono- and multi-) (Hayakawa et al., 1999; Gotoh et al., 2004; Smirnova et al., 2004; Ida et al., 
2005; Ida and Hayakawa, 2006), (3) principal component analysis (Gotoh et al., 2002) and (4) singular value decomposition (Hattori et al., 2006), and so on. Either when we are not sure about the presence of EQ-related ULF emissions or when we want to confirm the presence of EQ-related ULF emissions, the best way for this purpose is to apply different kinds of analysis procedures to the relevant ULF event and to compare and correlate the analysis results in order to make sure of the presence (or absence) of EQ-related ULF emissions.

In this paper we pay attention to a few relatively intense EQs that happened in China. We have already analyzed one particular EQ with magnitude 5.7, because we were lucky enough to have observed seismogenic ULF waves at a station close to the EQ epicenter (Ida et al., 2008). Here we will use the fractal analysis for the same and some other EQs in China, in order to confirm the presence of ULF emissions associated with the same EQ as already estimated by our previously improved polarization method. Finally, we discuss the observed fractal result in light of a few aspects (detectability of seismogenic emissions, comparison with the result by our previous analysis method, the importance of fractal analysis in the nonlinear process of the lithosphere and in EQ prediction).

\section{ULF geomagnetic data and EQs}

ULF geomagnetic data are obtained at an observatory named Kashi (geographic coordinates: $39.5^{\circ} \mathrm{N}, 76.0^{\circ} \mathrm{E}$ ) in China as indicated by a star in Fig. 1. At this field site we observe three geomagnetic components ( $H$ : NS component, $D$ : EW component, and $Z$ : vertical component) by means of a fluxgate magnetometer. The sampling frequency is $1 \mathrm{~Hz}$. The ULF data, since the commencement of observation at this station in the beginning of March 2003, are used for the analysis. So, the total period is $3 \mathrm{yr}$ and 10 months (nearly $4 \mathrm{yr}$ ) of 1 March 2003 through 31 December 2006.

There were observed several moderate EQs (with magnitude greater than 5.0 and close to 6.0) near the ULF station of Kashi: an EQ on 1 September $2003\left(M_{\mathrm{W}}=5.7\right)$, an EQ $\left(M_{\mathrm{W}}=5.4\right)$ on 26 September 2003, an EQ swarm in March 2003, the 4 May and 4 June 2003 EQs (these two happened nearly at the same place) and other EQs on 25 February 2005 and on 24 December 2006. The epicentral distance of the September $1 \mathrm{EQ}$ in 2003 is about $116 \mathrm{~km}$, that for the 26 September $2003 \sim 123 \mathrm{~km}$, and the epicentral distance of the right-hand EQs (4 May and 4 June) in Fig. 1 is about $100 \mathrm{~km}$. The epicentral distance of the 25 February $2005 \mathrm{EQ}$ is about $\sim 300 \mathrm{~km}$, whose EQ epicenter is located outside of Fig. 1. As is already noted by Hattori (2004) and Hayakawa et al. (2007), the epicentral distance of about $100 \mathrm{~km}$ is just around the threshold of ULF detection.

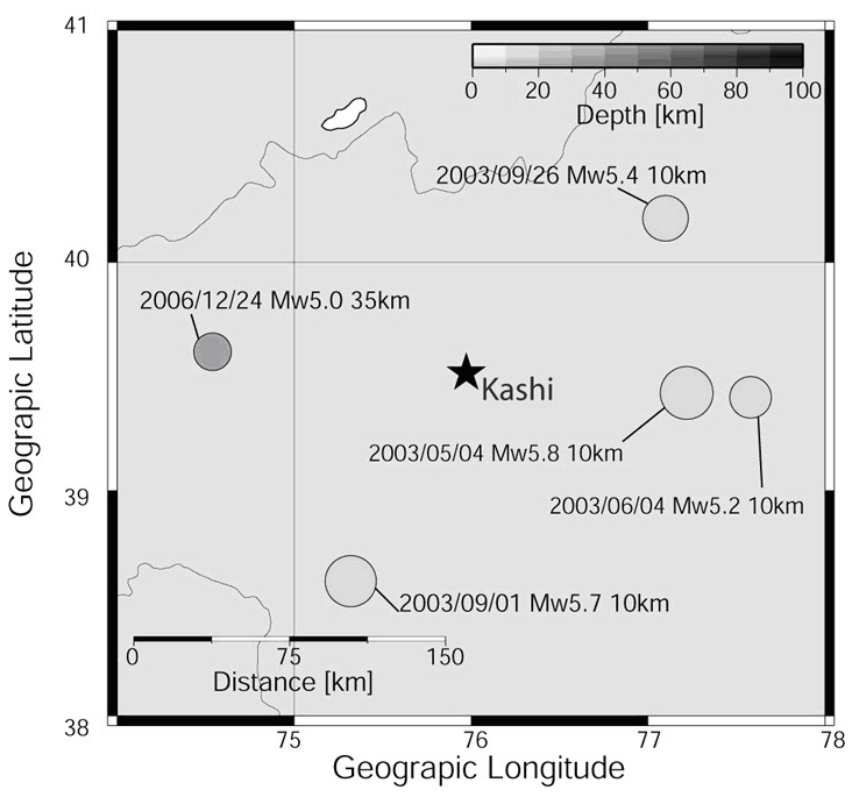

Fig. 1. The relative location of the ULF observation station (Kashi, a black star) and EQs (1 September, 26 September 2003; 4 May and 4 June 2003; 24 December 2006).

\section{Fractal analysis of ULF geomagnetic data}

Fractal analysis is known to be useful in elucidating the nonlinear process taking place in the lithosphere. Hayakawa et al. (1999) made the first attempt of fractal analysis of the ULF data for the 2003 Guam EQ event, and since then there has been an increased interest in this fractal analysis as applied to seismogenic emissions not only in the ULF frequency range (Smirnova et al., 2001, 2004; Gotoh et al., 2003, 2004; Ida et al., 2005; Ida and Hayakawa, 2006; Hayakawa and Ida, 2008), but also in different frequency ranges (DC, VHF etc.) (Eftaxias et al., 2004; Varotsos, 2005; Yonaiguchi et al., 2007).

We estimate the monofractal analysis for the ULF geomagnetic data sampled by $1 \mathrm{~s}$. Different kinds of methods to estimate the fractal dimension of any time-series data have been proposed so far: (1) the spectral slope of power frequency spectra (e.g. Hayakawa et al., 1999), (2) Burlaga and Klein method (Burlaga and Klein, 1986), and (3) Higuchi method (Higuchi, 1988). Gotoh et al. (2004) have compared these methods extensively by applying them to the test-data set of fractal Brownian motion with white noise and have concluded that the Higuchi method is the most accurate as compared with other methods. This the reason why we use this Higuchi method here to estimate the fractal dimension. The Higuchi method (Higuchi, 1998) is a kind of generalized curve length estimation. We calculate the length of our signal for different scales and find the dependence of the length on the scale. Let us describe the procedure. 
Initially, we have the time series data $X(i)(i=1, \ldots, N)$. For any given time scale $s$, we can construct a family of $s$ subseries $X_{m}(i ; s)$ of length $[(N-m) / s]([a / b]$ is the aliquot of division $a / b)$ :

$X_{m}(i ; s)=\{X(m+i s)\}, 1 \leq m \leq s$.

The curve length of each subseries is

$L_{m}(s)=\frac{1}{s} \sum_{i=1}^{\left[\frac{N-m}{s}\right]}|X(m+i s)-X(m+(i-1) s)| \frac{N-1}{s\left[\frac{N-m}{s}\right]}$.

And the average curve length of the family is given by

$L(s)=\frac{1}{s} \sum_{m=1}^{s} L_{m}(s)$

So, now we have obtained the dependence of the curve length $L(s)$ on the scale $s$. And if our signal has fractal properties, it will be possible to approximate $L(s)$ by a power function:

$L(s) \propto s^{-D_{\mathrm{f}}}$,

where $D_{\mathrm{f}}$ is the fractal dimension of the signal. The curve fitting of Eq. (8) can be estimated by the cross-correlation function of the measurement values and the regression line. When this coefficient is larger than 0.95 , the time-series data can be said to be fractal. The details of how to estimate the fractal dimension of any time-series data are given in Gotoh et al. (2003, 2004) and Ida and Hayakawa (2006).

\section{The results on fractal dimension}

The data during local nighttime ( $\mathrm{LT}=02: 00-06: 00)$ are used for analysis because of low local artificial noise at night, and one value is estimated for one night. We do not use wideband data, but the data are filtered just around the frequency of $0.01 \mathrm{~Hz}$ (period $=2 \mathrm{~s}$ to $20 \mathrm{~s}$ ) because this frequency is found to be a key frequency of seismogenic ULF emissions (Hayakawa et al., 2007). The temporal evolution of fractal dimension during the whole period is illustrated in Fig. 2. The top panel illustrates the temporal evolution of two different kinds of geomagnetic activity (upper, Dst index and lower, $\Sigma K_{\mathrm{p}}$ (daily sum of $K_{\mathrm{p}}$ index)). The lower three panels refer to the results for $Z$ component, $D$ component, and $H$ component, respectively. The black line in each panel indicates the temporal evolution of fractal dimension for each component ( $Z$ component is sensitive to the lithospheric activity), and the red line indicates the correlation coefficient in estimating the fractal exponent in the curve of $L(m) \propto m^{-D}$ (where $L(m)$ is the length of the curve)(the value of correlation is noted as the coefficient of determination on the right). The time of each EQ is indicated on the top of the figure. The total period of Fig. 2 is nearly $4 \mathrm{yr}$ from March 2003 to the end of 2006.

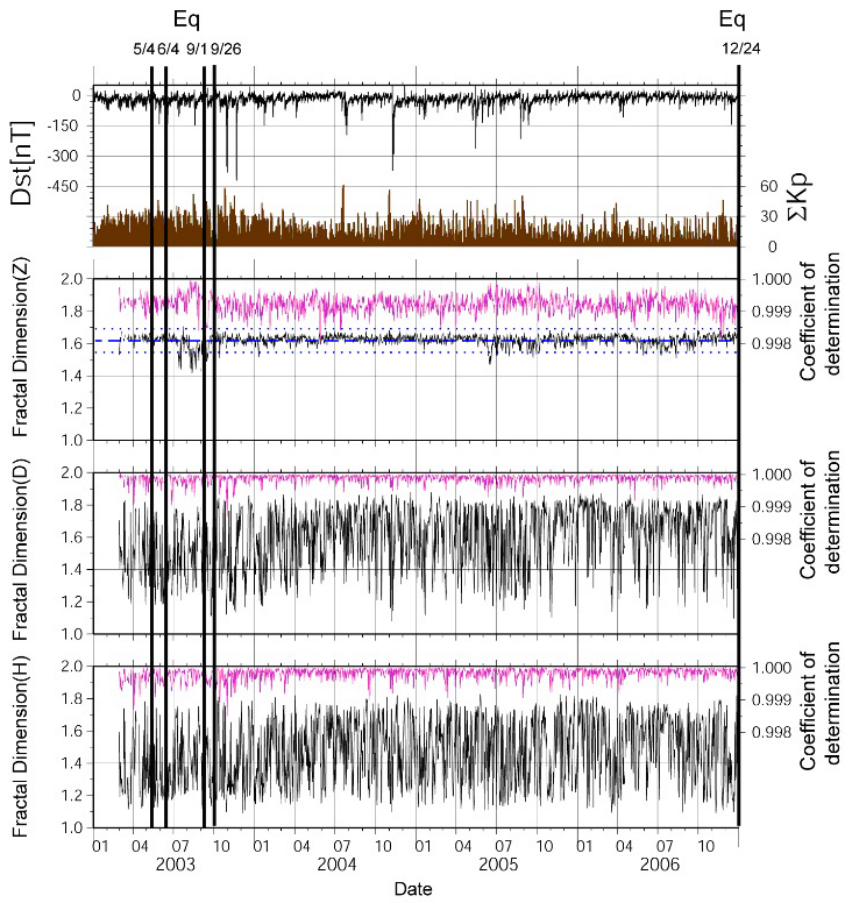

Fig. 2. Temporal evolutions of (top) geomagnetic activity (upper is $\operatorname{Dst}(n T)$ and the lower, $\left.\Sigma K_{\mathrm{p}}\right)$ and (bottom) fractal dimension (upper) and the coefficient of determination in fractal dimension analysis (lower). The data are from March 2003 to end of October of 2006, and the date of EQ is 1 September 2003. Horizontal blue broken line is the average value of fractal dimension during the whole period, while the upper and lower blue dotted lines mean $\pm 2 \sigma$ ( $\sigma$ : standard deviation) criteria.

The data length is long enough to find any significant changes in the fractal dimension of each component in Fig. 2. First of all, the fractal dimensions of two horizontal components $(H$ and $D)$ are found to fluctuate a lot, which enables us to detect no distinct changes in the temporal evolutions. On the contrary, we find a rather stable temporal evolution of fractal dimension of $Z$ component in the second panel. After looking at the data during the whole period of about $4 \mathrm{yr}$, the average value of fractal dimension of $Z$ component (indicated by a horizontal blue broken line) is just around 1.6, and we can notice only a few anomalous behaviours in the fractal dimension of $Z$ components (the value exceeding the $-2 \sigma(\sigma$ : standard deviation) line (indicated by a blue dotted line)): (1) a series of depletions in July and August 2003, (2) a sharp depletion in January 2004, and (3) a conspicuous minimum is June 2005 . Since there exist only 3 occasions of notable changes in the fractal dimension, we will discuss them one by one.

We first discuss the significant changes in fractal dimension of the $Z$ component during a few months from July to September 2003. The results during several months, encompassing the 1 September EQ, are presented in a new figure, Fig. 3, exactly in the same format as in Fig. 2. On the top of 


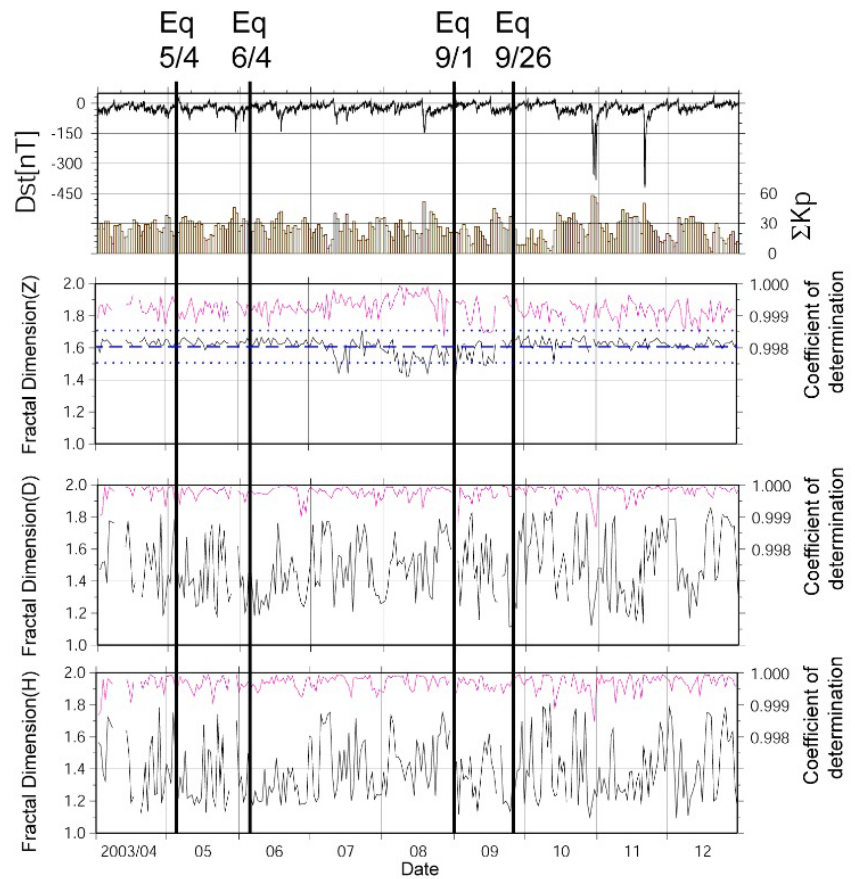

Fig. 3. The same as Fig. 2, but for a limited time period of April to December 2003, in order to find the fractal change for several EQs.

the figure, there have been indicated the dates of nearby EQs; there are four EQs with magnitude greater than 5.0. From the first glance at this figure, we find a very conspicuous and prolonged change in fractal dimension around the date of the 1 September EQ with magnitude of 5.8. The change (or decrease) in fractal dimension begins to appear around 10 July, recovers to the normal value at the end of July, and follows by a significant decrease during August. However, the tendency of decrease in the fractal dimension seems to be still taking place even after the EQ toward the end of September. Though we observe a rather small geomagnetic storm in the middle of August as seen from the Dst index, the change in fractal dimension started well before this geomagnetic storm, so that the change observed is unlikely to be connected with the geomagnetic storm and is highly likely to be associated with this EQ. Furthermore, as you can see from the bottom, the coefficient of determination in estimating the fractal dimension is found to be well enhanced when the fractal dimension is decreased. There is another EQ on 26 September, so that it is not so clear that a significant change in fractal dimension from the beginning of September to 20 September is a precursor to this $9 / 26 \mathrm{EQ}$, or the after-effect of the previous 9/1 EQ. On the contrary, it seems that there is definitely no response in the fractal dimension to other EQs on 4 May or 4 June 2003, as seen in Fig. 3.

The second one takes place as a sharp depletion in the fractal dimension in January 2004, which is highly likely to be due a sharp variation in geomagnetic activity as a geomagnetic storm. Because Mezentsev et al. (2009) have analyzed the fractal dimension of ULF geomagnetic data at different stations and over long-term data, they have found that the geomagnetic storm tends to have a significant effect and that the fractal dimension always decreases for geomagnetic storms.

As for the third event in June 2005, we have investigated several possibilities. Initially, we have checked different parameters of space weathers including geomagnetic variations, but it is concluded that there is an extremely low probability of the solar-terrestrial effect. Then, we have checked EQs just around the observing station, which indicated that there were no EQs (even smaller magnitude) around the observing station. Then, we have checked the magnetic variations carefully, and it is found that the intensity of $Z$ components during this period is considerably enhanced as compared with other periods, which seems to be similar to the characteristics just before the EQ in March 2003. Finally, we cannot come to any definite conclusion on the origin of a significant change in fractal dimension for the 3rd period.

\section{Summary and conclusion}

The long-term data of nearly four years (since the commencement of ULF observation at Kashi in China) have been used to try to find any effect precursory to nearby EQs by means of the fractal analysis.

As the result of fractal analysis, it is found that no significant effects are detected for the horizontal ( $D$ and $H$ ) components, but some significant effects (as a decrease in fractal dimension) are observed only in the $Z$ component only for the EQs on 1 September $2003(M=5.7)$ and 26 September $2003(M=5.4)$. Other EQs with magnitude greater than 5.0 are found to induce no effect on the fractal dimension of $Z$ component. The biggest magnitude EQ on 4 May 2003 $(M=5.8)$ did not induce any change in fractal dimension.

The following summary can be reached from the present work.

1. When the epicentral distance is over $100 \mathrm{~km}$, it is generally difficult for us to detect any precursory effect in ULF signals for EQs with magnitude on the order of $M \geq 5$, even with using the fractal analysis.

2. For the biggest two EQs with magnitude $M_{\mathrm{w}}=5.7$ and 5.8 , we could find a significant change in fractal dimension for one EQ, but we could not for another one, even though the epicentral distances of those two EQs were nearly the same -105 and $113 \mathrm{~km}$.

3. It seems that the fractal analysis of $Z$ component is quite effective in finding any precursor for medium magnitude EQs with epicentral distance over $100 \mathrm{~km}$.

We will discuss the above experimental results in the context of a few physical aspects: (1) detectability of seismogenic ULF emissions, (2) comparison of this fractal results with our 
previous analysis by means of polarization analysis, (3) the importance of fractal analysis in nonlinear lithospheric effect and in EQ prediction.

\subsection{Detectability of seismogenic ULF emissions}

Hattori (2004), Hayakawa et al. (2007) and Molchanov and Hayakawa (2008) have summarized the previous ULF events during the last two decades, and come to the conclusion that seismogenic ULF emissions can be detectable with a radius of $100 \mathrm{~km}$ for $M=7.0$ and $70-80 \mathrm{~km}$ for $M=6.0$. By comparing the epicentral distance of $110-120 \mathrm{~km}$ for the two EQs with $M_{\mathrm{w}}=5.7$ and 5.8 with the above empirical threshold distance, it seems difficult for us to detect any precursory effects of ULF emissions for those EQs. However, we can conclude that the use of fractal analysis (especially for the $Z$ component) enables us to detect a precursory effect for some particular EQs with moderate magnitude (greater than 5.0, but less than 6.0). Two EQs had nearly the same magnitudes of $M=5.7$ and 5.8, and again with nearly the same epicentral distances. However, one EQ had an apparent ULF signature, but another had no signature. As mentioned before, the epicentral distances are over $100 \mathrm{~km}$, which is too far for the detection of seismogenic ULF emissions by means of simple analysis such as amplitude analysis. The most probable reason for this would be the radiation directivity of seismogenic ULF emissions depending on the relative direction between the generated current source and the receiving station, as already suggested by Molchanov et al. (1995), Tian and Hata (1996), Dong et al. (2005), and Bortnik et al. (2010). If the epicentral distance is not so large, the directivity issue does not have such a significant effect.

\subsection{Comparison of the fractal analysis result with our previous polarization analysis}

An improved polarization analysis has been applied to the same ULF data for the same period (Ida et al., 2008) by making full use of the ratio of the magnetic vertical to horizontal component. They have found an apparent increase only just before the EQ on 1 September 2003, so that our fractal analysis result in this paper is found to be consistent with the conclusion of Ida et al. (2008) and would provide a further support to the presence of precursory ULF emissions for this 1 September EQ. The common results by two different analysis results would enable us to conclude that a precursory ULF emission is definitely generated before this EQ.

\subsection{The importance of fractal analysis in the nonlinear process in the lithosphere}

Because we know that when a heterogeneous material is strained, its evolution toward rupture (or EQ) is characterized by the nucleation and coalescence of microcracks before the final break-up. ULF electromagnetic emissions are considered to be produced by such microcracks (e.g. Molchanov and Hayakawa, 1995, 2008), which can be considered as a precursor to the general rupture. Hayakawa and Ida (2008) have shown that the fractal analysis (both mono- and multifractal) would be of extreme use in studying such a nonlinear process taking place in the lithosphere.

In the present paper, we have found a significant decrease in fractal dimension of the $Z$ component exceeding the $2 \sigma$ criterion before the 1 September $2003 \mathrm{EQ}$. Though there have been published few papers on the fractal analysis of seismogenic ULF emissions (Hayakawa et al., 1999; Smirnova et al., 2003; Gotoh et al., 2004; Ida and Hayakawa, 2006), let us try to compare the result in this paper with our previous results. The first event is the 20003 Guam EQ (Hayakawa et al., 1999; Ida and Hyayakawa, 2006; Hayakawa and Ida, 2008), in which the monofractal dimension of seismogenic ULF emissions is found to increase before the Guam EQ, and this change is opposite to the result in this paper. The second is the 2000 Izu Islands EQ swarm (Gotoh et al., 2004), in which the monofractal dimension exhibited again a significant increase for this EQ swarm. The important point is that the fractal dimension of the $Z$ component on the normal days (non-seismic period) is just around $1.5-1.6$ for all the events, and we can anticipate a significant change in fractal dimension: an increase for some EQs (Guam and Izu islands) and a decrease for other EQs (in this paper). We could find a precursory decrease in the fractal dimension before one definite case of 1 September $2003 \mathrm{EQ}$, which would suggest that the nonlinear process is really taking place in the lithosphere during two months before the EQ and would be a precursor of EQ prediction. Any change in fractal dimension (either an increase in the case of the Guam and Izu EQs and a decrease in the present case) before the EQ might be related to the lithospheric change; in other words, it might offer the information on the generation mechanism of ULF emissions.

Though our target is seismogenic ULF emissions, it would be of great use to pay attention to the studies of acoustic emissions (reflecting the microfracturing process). There have been published several papers on the changes in acoustic emissions when loading pressure to the rock in the laboratory. Ponomarev et al. (1997) found a significant decrease in fractal dimension just before the global instability in the laboratory experiment. Then, the spatial distribution of acoustic emissions of rock fracture experiment was used to obtain a decrease in fractal dimension before the rupture (Jaeger and Cook, 1976; Hirata et al., 1987). The temporal evolution of acoustic emissions was also studied by Hirata (1987), who found that the temporal evolution of acoustic emissions becomes fractal over a range from microfracture to an EQ.

While the fractality of EQ patterns (spatial and temporal) has been firmly established (e.g. Goltz, 1997), there have been few reports on the use of fractal spectral change as a precursor to EQs (Jiang, 1993; Hirabayashi et al., 1992). Jiang (1993) was interested in the change of multi-fractal parameters for moderately large EQs, and he concluded that the 
temporal variations in some of multi-fractal parameters provide some message for medium-term forecast of EQs. Then, the main focus of the paper by Hirabayashi et al. (1992) was on showing that seismicity from three seismo-active regions (California, Greece, and Eastern Japan) constitutes multi-fractal measures, and they showed some precursory behaviour. The similar work to these has been recently published by Kiyaschenko et al. (2003) in order to find some medium-term EQ precursors. The epicentral distributions analysed by these authors are known to be useful for the medium-term prediction, but the short-term EQ prediction seems to be possible only with the use of electromagnetic phenomena.

It is confirmed that the acoustic emissions exhibit a distinct decrease in the fractal dimension of acoustic emissions. We believe that the acoustic emission reflects the mechanical process in the rock (especially the generation of cracks or microfracturing), but it is quite uncertain how this microfracture is related with the generation of seismogenic ULF emissions. In other words, since the microfracture is just a mechanical effect, the most important point is how to have electrification due to this microfracture; this is the generation mechanism of seismogenic ULF emissions. As is summarized in Molchanov and Hayakawa (2008), there have been proposed a few mechanisms, including the electrokinetic effect (e.g. Mizutani et al., 1976), piezo effect (e.g. Nitsan, 1997), positive-hole effect (Freund, 2009), microfracturing hypothesis (Molchanov and Hayakawa, 1995) and so on. Here we try to connect the change in fractal dimension of seismogenic ULF emissions with these generation mechanisms. In the case of the 2003 Guam EQ and the 200 Izu islands EQ swarm, we have observed an increase of fractal dimension before an EQ, while we have observed a completely opposite result of a significant decrease in fractal dimension for an EQ in China as in this paper. We here connect the change in fractal dimension simply to the change in frequency spectrum of ULF emissions generated. An increase in fractal dimension may indicate that we expect the dominance in higher frequency range even in the ULF range, while a decrease in fractal dimension may be related to the dominance of lower frequency components. So, the decrease in the fractal dimension in this paper for an inland EQ in China may be hypothesized to be due to such a slow process as the electro-kinetic effect or so. We may expect the higher-frequency dominated mechanism like the microfracturing hypothesis for the cases of an increase in fractal dimension as for the Guam and Izu EQs. Of course, further details are highly required in future.

Acknowledgements. One of the authors (MH) is grateful to Mayekawa Houonkai Foundation for its support.

Edited by: A. G. Hunt

Reviewed by: N. Gershenzon, A. P. Nickolaenko, and two anonymous referees

\section{References}

Bortnik, J., Bleier, T. E., Dunson, C., and Freund, F.: Estimating the seismotelluric current required for observable electromagnetic ground signals, Ann. Geophys., 28, 1615-1624, doi:10.5194/angeo-28-1615-2010, 2010.

Burlaga, L. F. and Klein, L. W.: Fractal structure of the interplanetary magnetic field, J. Geophys. Res., 91, 347-350, 1986.

Campbell, W.: Natural magnetic disturbance fields, not precursors, preceding the Loma Prieta earthquake, J. Geophys. Res., 114, A05307, doi:10.1029/2008JA013932, 2009.

Dong, J., Gao, Y., and Hayakawa, M.: Analysis on subaerial electric field radiated by a unit electric current source in the ground, IEEJ (Inst. Elecr. Engrs. Japan), Trans. Fundament. Materials, 125, 591-595, 2005.

Eftaxias, K., Frangos, P., Kapiris, P., Polygiannakis, J., Kopanas, J., Peratzakis, A., Skountzos, P., and Jaggard, D.: Review-model of pre-seismic electromagnetic emissions in terms of fractal electrodynamics, Fractals, 12, 243-273, 2004.

Fraser-Smith, A. C.: The ultralow-frequency magnetic fields associated with and preceding earthquakes, in: Electromagnetic Phenomena Associated with Earthquakes, edited by: Hayakawa, M. Transworld Research Network, Trivandrum, India, 1-20, 2009.

Fraser-Smith, A. C., Bernardi, A., McGill, P. R., Ladd, M. E., Helliwell, R. A., and Villard Jr., O. G.: Low-frequency magnetic field measurements near the epicenter of the Ms 7.1 Loma Prieta earthquake, Geophys. Res. Lett., 17, 1465-1468, 1990.

Freund, F.: Stress-activated positive hole charge carriers in rocks and the generation of pre-earthquake signals, in: Electromagnetic Phenomena Associated with Earthquakes, edited by: Hayakawa, M., Transworld Research Network, Trivandrum India, 41-96, 2009.

Goltz, C.: Fractal and Chaotic Properties of Earthquakes, Springer Berlin, 178 pp., 1997.

Gotoh, K., Akinaga, Y., Hayakawa, M., and Hattori, K.: Principal component analysis of ULF geomagnetic data for Izu islands earthquakes in July 2000, J. Atmos. Electr., 22, 1-12, 2002.

Gotoh, K., Hayakawa, M., and Smirnova, N.: Fractal analysis of the ULF geomagnetic data obtained at Izu Peninsula, Japan in relation to the nearby earthquake swarm of June-August 2000, Nat. Hazards Earth Syst. Sci., 3, 229-236, doi:10.5194/nhess-3229-2003, 2003.

Gotoh, K., Hayakawa, M., Smirnova, N. A., and Hattori, K.: Fractal analysis of seismogenic ULF emissions, Phys. Chem. Earth, 29, 419-424, 2004.

Hattori, K.: ULF geomagnetic changes associated with large earthquakes, Terr. Atm. Ocean Sci., 15, 329-360, 2004, http://www.ocean-sci.net/15/329/2004/.

Hattori, K., Serita, A., Yoshino, C., Hayakawa, M., and Isezaki, N.: Singular spectral analysis and principal component analysis for signal discrimination of ULF geomagnetic data associated with 2000 Izu Island earthquake swarm, Phys. Chem. Earth, 31, 281291, 2006.

Hayakawa, M. (Ed.): Electromagnetic Phenomena Associated with Earthquakes, Transworld Research Network, Trivandrum, India, 279 pp., 2009.

Hayakawa, M. and Ida, Y.: Fractal (mono- and multi-) analysis for the ULF data during the 1993 Guam earthquake for the study of prefracture criticality, Curr. Develop. Theory Appl. Wavelets, 2, 159-174, 2008. 
Hayakawa, M. and Molchanov, O. A. (Eds.): Seismo Electromagnetics: Lithosphere - Atmosphere - Ionosphere Coupling, TERRAPUB, Tokyo, Japan, 477 pp., 2002.

Hayakawa, M., Kawate, R., Molchanov, O. A., and Yumoto, K.: Results of ultra-low-frequency magnetic field measurements during the Guam earthquake of 8 August 1993, Geophys. Res. Lett., 23, 241-244, 1996.

Hayakawa, M., Itoh, T., and Smirnova, N.: Fractal analysis of ULF geomagnetic data associated with the Guam earthquake on $8 \mathrm{Au}$ gust 1993, Geophys. Res. Lett., 26, 2797-2800, 1999.

Hayakawa, M., Hattori, K., and Ohta, K.: Monitoring of ULF (ultralow-frequency) geomagnetic variations associated with earthquakes, Sensors, 7, 1108-1122, 2007.

Higuchi, T.: Approach to an irregular time on the basis of fractal theory, Physica D, 31, 277-283, 1988.

Hirabayashi, Y., Ito, K., and Yoshii, T.: Multifractal analysis of earthquakes, Pageoph, 12, 591-610, 1992.

Hirata, T.: Omori's power law aftershock sequences of microfracturing in rock fracture experiment, J. Geophys. Res., 92, 62156221, 1987.

Hirata, T., Satoh, T., and Ito, K.: Fractal structure of spatial distribution of microfracturing in rock, Geophys. J. Royal Astronom. Soc., 90, 369-374, 1987.

Ida, Y. and Hayakawa, M.: Fractal analysis for the ULF data during the 1993 Guam earthquake to study prefracture criticality, Nonlin. Processes Geophys., 13, 409-412, doi:10.5194/npg-13409-2006, 2006.

Ida, Y., Hayakawa, M., Adalev, A., and Gotoh, K.: Multifractal analysis for the ULF geomagnetic data during the 1993 Guam earthquake, Nonlin. Processes Geophys., 12, 157-162, doi:10.5194/npg-12-157-2005, 2005.

Ida, Y., Yang, D., Li, Q., Sun, H., and Hayakawa, M.: Detection of ULF electromagnetic emissions as a precursor to an earthquake in China with an improved polarization analysis, Nat. Hazards Earth Syst. Sci., 8, 775-777, doi:10.5194/nhess-8-7752008, 2008.

Jaeger, J. C. and Cook, N. G. W.: Fundamental of Rock Mechanics, Chapman and Hall, London, 1976.

Jiang, H. K.: The multifractal local scaling feature of "spatial energy generating" and its seismic precursory information, Int'l Symp. on Fractal and Dynamic Systems in Geoscience, Book of Abstracts, vol. 1, 12 pp., Frankfurt, April 1993.

Kiyashchenko, D., Smirnova, N., Troyan, V., and Vallianatos, F.: Dynamics of multifractal and correlation characteristics of the spatio-temporal distribution of regional seismicity before the strong earthquakes, Nat. Hazards Earth Syst. Sci., 3, 285-298, doi:10.5194/nhess-3-285-2003, 2003.

Kopytenko, Y. U. A., Matiashvili, T. G., Veronov, P. M., and Molchanov, O. A.: Detection of ultra-low frequency emissions connected with the Spitak earthquake and its aftershock activity, based on geomagnetic pulsation data at Dusheti and Vardzia, Phys. Earth Planet. Int., 77, 85-95, 1993.

Mezentsev, A. Yu., Hayakawa, M., and Hattori, K.: Fractal ULF signatures related to seismic processes, J. Atmos. Electr., 29, 8193, 2009.
Mizutani, H., Ishido, T., Yokokura, T., and Ohnishi, S.: Electrokinetic phenomena associated with earthquakes, Geophys. Res. Lett., 3, 365-368, 1976.

Molchanov, O. A. and Hayakawa, M.: Generation of ULF electromagnetic emissions by microfracturing, Geophys. Res. Lett., 22, 3091-3094, 1995.

Molchanov, O. A. and Hayakawa, M.: Seismo-Electromagnetics and Related Phenomena, History and latest results, TERRAPUB, Tokyo, Japan, 189 pp., 2008.

Molchanov, O. A., Kopytenko, Yu. A., Voronov, P. V., Kopytenko, E. A., Matiashvili, T. G., Fraser-Smith, A. C., and Bernardi, A.: Results of ULF magnetic field measurements near the epicenters of the Spitak $\left(\mathrm{M}_{s}=6.9\right)$ and the Loma Prieta $\left(\mathrm{M}_{s}=7.1\right)$ earthquakes: Comparative analysis, Geophys. Res. Lett., 19, 14951498, 1992.

Molchanov O. A., Hayakwa, M., and Rafalsky, V. A.: Penetration characteristics of electromagnetic emissions from an underground seismic source into the atmosphere, ionosphere, and magnetosphere, J. Geophys. Res., 100, 1691-1712, 1995.

Moore, G. W.: Magnetic disturbances preceding the 1964 Alaska earthquake, Nature, 203, 508-509, 1964.

Nitsan, U.: Electromagnetic emission accompanying fracture of quartz-bearing rocks, Geophys. Res. Lett., 4, 333-336, 1977.

Ponomarev, A., Zavyalov, A., Smirnov, V., and Lockner, D.: Physical modeling of the formation and evolution of seismically active fault zones, Tectonophys., 277, 57-81, 1997.

Smirnova, N., Hayakawa, M., Gotoh, K., and Volobuev, D.: Scaling characteristics of ULF geomagnetic fields at the Guam seismoactive area and their dynamics in relation to the earthquake, Nat. Hazards Earth Syst. Sci., 1, 119-126, doi:10.5194/nhess-1-1192001, 2001.

Smirnova, N., Hayakawa, M., and Gotoh, K.: Precursory behavior of fractal characteristics of the ULF electromagnetic fields in seismic active zones before strong earthquakes, Phys. Chem. Earth, 29, 445-451, 2004.

Thomas, J. N., Love, J. J., Johnston, M. J. S., and Yumoto, K.: On the reported magnetic precursor of the 1993 Guam earthquake, Geophys. Res. Lett., 36, L16301, doi:10.1029/2009GL039020, 2009a.

Thomas, J. N., Love, J. J., and Johnston, M. J. S.: On the reported magnetic precursor of the 1989 Loma Prieta earthquake, Phys. Earth Planet. Int., 173, 207-215, 2009b.

Tian, X. and Hata, M.: Analysis of seismogenic radiation and transmission mechanisms, J. Atmos. Electr., Special issue on Seismoelectromagnetic phenomena, edited by: Hayakawa, M., 16, $227-$ 235, 1996.

Varotsos, P., The Physics of Seismic Electric Signals, TERRAPUB, Tokyo, 338 pp., 2005.

Yonaiguchi, N., Ida, Y., Hayakawa, M., and Masuda, S.: Fractal analysis for VHF electromagnetic noises and the identification of preseismic signature of an earthquake, J. Atmos. Sol.-Terr. Phys., 69, 1825-1832, 2007. 